\title{
Outcomes of Osteoarthritis after Bariatric Surgery
}

\section{Lan Vư ${ }^{1}$, Noah J. Switzer ${ }^{2}$, D’Arcy C. Durand ${ }^{1}$, Douglas Hedden ${ }^{1,3}$ and Shahzeer Karmali1,3*}

${ }^{1}$ Department of Surgery, University of Alberta, Edmonton, Alberta, Canada

${ }^{2}$ Faculty of Medicine \& Dentistry, University of Alberta, Edmonton, Alberta, Canada

${ }^{3}$ Department of Surgery, University of Alberta, Center for the Advancement of Minimally Invasive Surgery (CAMIS), Edmonton, Alberta, Canada

\begin{abstract}
Joint Osteoarthritis $(\mathrm{OA})$ is a major source of morbidity and disability in the aging population. It is especially a concern to overweight individuals, as obesity is an important risk factor for the development of OA. The relationship between obesity and the development of joint damage is not purely the result of mechanical forces as pathologic inflammatory markers have been implicated in the process as well. Recent clinical practise guidelines state that weight-loss leads to functional improvement in joint pain. It can then be expected that bariatric surgery, the only proven long-term weight-loss modality in the morbidly obese, should have a similar effect. However, the improvement in OA post-surgery is not as drastic in comparison to the change in other obesity-related co-morbidities. Bariatric surgery, however, can be used in conjunction with orthopaedic surgery to ultimately treat OA through joint replacement. Effective weight-loss, as achieved through bariatric surgery, can improve post-operative outcomes in the obese population, making poor surgical candidates into acceptable ones. In this editorial, we explore the relationship between obesity and osteoarthritis and the roles of combining bariatric and orthopaedic surgeries.
\end{abstract}

Keywords: Osteoarthritis; Bariatric surgery, Outcomes in osteoarthritis

\section{Introduction}

Obesity affects a growing number of North Americans. In the United States alone $1 / 3$ of all adults are classified as obese [1]. Body Mass Index (BMI) measurements are used to categorize patients into different severities of obesity. A BMI of less than $25 \mathrm{~kg} / \mathrm{m}^{2}$ is considered normal. Patients are classified as overweight with a BMI between 25 and $30 \mathrm{~kg} / \mathrm{m}^{2}$, obese with a BMI that is over $30 \mathrm{~kg} / \mathrm{m}^{2}$, and morbidly obese with a BMI that is over $40 \mathrm{~kg} / \mathrm{m}^{2}$. Bariatric surgery has been shown to be the most effective treatment modality for achieving significant weight loss in the morbidly obese [2]. Morbid obesity is associated with a number of conditions that considerably affect the patient's health $[1,3]$. These conditions include diabetes, hypertension, dyslipidemia, obstructive sleep apnea, gastroesophageal reflux disease, depression, as well as joint pain in the knees and lower back [4]. Along with achieving clinically significant excess weight-loss, bariatric surgery has also shown effectiveness in improving many of these aforementioned co morbid conditions [3]. In particular, type 2 diabetes and hypertension resolve in the overwhelming majority of patients undergoing bariatric surgery $[3,5,6]$. The effect of bariatric surgery on osteoarthritis and pain in weight-bearing joints, however, is not as clear.

Osteoarthritis (OA) is known to be exacerbated or possibly even caused by obesity. This relationship is not as simple as originally postulated, as it is not just the increased weight exerted on the joints that accounts for the development of osteoarthritis [7]. It is this complexity that complicates the relationship between bariatric surgery and the progression of osteoarthritis in weight-bearing joints. In a recent systematic review, we have found that there is a paucity of data in the current literature establishing a clear relationship between bariatric surgery and the progression or cessation of osteoarthritis [7]. However, we found that the available studies do support the notion that there is a relationship between improved joint pain after significant weight loss following bariatric surgery [7]. The purpose of this review is to provide health practitioners with an update and review on the impact and effect of bariatric surgery on osteoarthritis.

\section{Discussion}

\section{Obesity as a cause of osteoarthritis}

The two main risk factors for the development of osteoarthritis are advanced age and being overweight. Although age is the most important risk factor, obesity is the greatest modifiable risk factor. This increased risk is felt to be particularly high in weight-bearing joints [8]. This is supported by the Framingham Study where obese patients were found to have a $50 \%$ increased risk of knee OA when compared to controls [9]. Moreover, the relationship between obesity and knee OA has been further linked by a meta-analysis of 36 papers which found a strong correlation between knee OA and obesity $[10,11]$. In another review of the literature, patients with a BMI $>30$ were found to have a 7 fold increased risk for developing knee $\mathrm{OA}$ as compared to patients with a $\mathrm{BMI}<25[11,12]$. However, the evidence is not as strong regarding the interplay between obesity and OA in other weight-bearing joints such as the hip and ankle $[8,13,14]$. Although obese individuals were found to have a higher risk for back pain than non-obese individuals $(58 \%$ compared to $24 \%$ ), it is felt that back pain is only moderately associated with obesity $[8,15]$. Furthermore, it is difficult to differentiate the aetiology of back pain since many factors may be involved; thereby complicating studies investigating back OA.

These discrepancies suggest that there are more than simple mechanical forces in the development of osteoarthritis. It is felt that important biochemical factors are also altered in obese patients, which may accelerate the development of $\mathrm{OA}$ in certain joints. Local inflammatory factors, such as IL-1, IL-6, CRP, and TNF-alpha have been shown to be elevated in obese patients [16-18]. Furthermore, inflammation is also mediated by adiponectin and leptin, which contribute to cartilage breakdown $[17,19]$. Finally, the muscle weakness that accompanies obesity, particularly in the quadriceps and lumbar muscle groups, contributes to the progression of $\mathrm{OA}$ in the knees as a result of altered function, biomechanics, and gait patterns [17].

*Corresponding author: Shahzeer Karmali, Royal Alexandra Hospital, Room 405, Community Services Center, 10240 Kingsway, Edmonton, Alberta, Canada, T5H 3V9, Tel : 780-735-6650; Fax: 780-735-6652; E-mail: shahzeer@ualberta.ca

Received April 26, 2013; Accepted August 22, 2013; Published August 29, 2013

Citation: Vu L, Switzer NJ, Durand DAC, Hedden D, Karmali S (2013) Outcomes of Osteoarthritis after Bariatric Surgery. Surgery Curr Res 3: 141. doi:10.4172/21611076.1000141

Copyright: ( 2013 Vu L, et al. This is an open-access article distributed under the terms of the Creative Commons Attribution License, which permits unrestricted use, distribution, and reproduction in any medium, provided the original author and source are credited. 
Overall, obesity is strongly associated with the development of knee $\mathrm{OA}$ and moderately associated with lower back OA. Its relationship with other weight-bearing joints, such as the hip, is not clear. The contributing factors consist not only of the increased mechanical load, but also involve humoral and inflammatory factors.

\section{Weight loss relieving osteoarthritis}

As obesity is considered to be the most significant modifiable cause of osteoarthritis, studies have been designed to prove that weight loss should lead to an expected improvement or possible prevention of OA. Surprisingly, there are very few studies that have succeeded in demonstrating the relationship between conventional weight loss and OA. This is due primarily to the difficulty in maintaining uniform weight loss in a study group through conventional means such as diet, exercise and medication. An often cited randomized control trial by Christensen et al., randomized 80 patients to a low-energy diet and a normal diet for 8 weeks [20]. The low-energy diet wing was found to have improved Western Ontario/McMaster (WOMAC) scores, scores which assess functionality in patients with OA [20]. The authors extrapolated that a $10 \%$ weight reduction corresponded to a $28 \%$ improvement in function, as measured by WOMAC. This finding was supported by a meta-analysis which showed moderate to large clinical improvements in joint pain [20] However, when dealing with a chronic disease, long-term follow-up is required to adequately assess the relationship between weight-loss and improvement in OA, yet this study only had short-term data. Another randomized trial, assessing the effect of diet and exercise on WOMAC scores, found similar results and determined that a $5 \%$ weight loss over 18 months resulted in a $24 \%$ improvement in function $[18,21]$. However, this study was limited by the high rate of patients lost to follow-up (20\%). Other studies have attempted to examine the functional outcomes of medically induced weight-loss, but these studies were also hampered by non-adherence to the medication and by weight recidivism $[18,22]$.

Brosseau et al. [23] recently published an updated clinical practice guideline examining the effect of weight-loss and osteoarthritis. Although they found the quality of most studies to be methodologically low, they were able to establish a consensus that physical activity and diet programs are beneficial to relieve pain and to improve functional status in patients with OA.

\section{Bariatric Surgery link to improved osteoarthritis}

There have been numerous studies examining the effect of bariatric surgery on the progression of osteoarthritis. An early study by McGoey et al. [24] demonstrated an improvement in lumbar spine pain from 62 to $11 \%$, hip pain from $11 \%$ to $2 \%$ and knee pain from 57 to $14 \%$ in 62 patients who underwent roux-en-Y gastric bypass (RYGB). A later study by Hooper et al. published similar results in a prospective study of 48 patients with joint pain who underwent RYGB [8]. Patients experienced an average weight loss of $41 \mathrm{~kg}$, coinciding to a BMI reduction from 51 to 36 , and a significant improvement in pain in weight-bearing joints from $100 \%$ to $37 \%$ postoperatively. Interestingly, there was also a similar reduction in pain scores from $79 \%$ to $40 \%$ in non-weight-bearing joints and McGoey et al. found that there was no difference in pain reduction between patients who lost $<60 \mathrm{lbs}(27$ $\mathrm{kg})$ compared to patients who lost $>99 \mathrm{lbs}(45 \mathrm{~kg})[8,24]$. These two observations lend credence to the hypothesis that factors other than mechanical loads contribute to the development of OA. The authors also postulate that a psychosomatic component could contribute to ameliorate symptoms as pain is a subjective variable. Thus, any somatised pain could have been improved in this group of motivated patients.
Peltonen et al. [25] also demonstrated an improvement in workrelated restricting pain. In a large case-controlled study, surgical management of obesity was compared to conventionally managed controls. The authors reported that surgical intervention accounted for an average weight loss of $28 \mathrm{~kg}$ with significant improvement in workrelated pain in five different locations (neck back, hip, knee, and ankle).

However, other studies have failed to support this conclusion. Korenkov et al. [26] followed 145 patients over 8 years who underwent adjustable gastric band. Despite a BMI drop from 48 to 34 and an improvement of other medical comorbidities, they only reported a drop in knee pain from $47 \%$ to $38 \%$. Although a statistically significant result, the clinical difference was quite minimal. It was postulated that there was a less than expected effect on knee pain because degeneration had already occurred and thus the damage was irreparable [26].

Thus, it can then be extrapolated that it is important to alter the course of OA before joint changes become permanent. Therefore, there may be a role for bariatric surgery in preventative healthcare. An interesting study by Abu-Abeid et al. [27] studied 64 patients with knee pain and early signs of OA on radiographs, who underwent bariatric surgery. At 3-month follow-up, the joint space increased an average of $0.65 \mathrm{~mm}$ with an associated average BMI reduction of $6.3 \mathrm{~kg} / \mathrm{m}^{2}$ which correlated with improved pain-control. In fact, a larger change in BMI correlated with a larger joint space.

A recent systematic review examining the effect that bariatric surgery has on hip and knee osteoarthritis was conducted by our group [7]. Among 400 related articles, only six studies were found to be appropriate for analysis. Of these six studies, five were case series and only one was a case-controlled study. We concluded that bariatric surgery does confer a moderate improvement in both Knee Society Score (KSS) and Harris Hip Score, however this improvement is tempered compared to the improvement seen in other comorbid conditions such as diabetes and hypertension post-surgery [3,7]. Still further prospective studies with a larger patient population are needed to be fully comfortable in describing the effect that bariatric surgery has on OA [7].

The small variation in effect that is seen between studies is most likely a result of osteoarthritis being affected by many different factors. Mechanical causes, as well as humoral and inflammatory causes play a role in the degeneration. Furthermore, many of the studies focus on pain relief, which is a subjective entity. This subjectivity may or may not be affected by the improvement in mental depression and activity that accompanies many patients who undergo bariatric surgery, regardless of the presence of OA $[18,28]$

\section{Outcomes of weight loss prior to orthopaedic surgery}

As the eventual treatment for osteoarthritis is joint replacement, there has been interest directed at the management of obesity prior to orthopaedic surgery and whether this affects patient outcomes. Morbid obesity is considered a relative contraindication to unilateral knee replacement $[18,29]$. Obese patients have higher rates of wound infection, dehiscence, venous thromboembolic events, prosthetic loosening and cardiac complications after arthroplasty [30,31]. Moreover, Foran et al. showed that a BMI $>30$ had a negative effect on outcomes in Total Knee Replacements (TKR) [29]. To add to this, resource utilization for obese patients increased on average by $7-9 \%$ compared to non-obese patients who have hip or knee replacements [18].

Studies examining outcome measures of weight-loss prior to joint replacement have been very difficult to conduct due to the ineffectiveness 
of conventional weight-loss measures in attaining significant and sustained weight loss. The inability of these patients to mobilize is cited as the main reason for weight-loss failure. A questionnaire by Howarth et al. [32] examined 45 obese patients awaiting orthopaedic operations [32]. They found that although half of the patients felt it was important to lose weight prior to surgery, and despite $89 \%$ of patients having made an attempt to lose weight, up to $55 \%$ of patients were unable to lose the weight that they had expected to lose and $25 \%$ were unable to maintain the weight loss. The study cited that lack of motivation accounted for a greater part of reason than knee pain did for inability to lose weight. In addition, on average, patients do not lose weight post total joint replacement as there is a high correlation between pre- and post-operative BMI $[33,34]$. Thus, patients should not rely on total joint replacement as a method of weight loss as physical activity levels are not increased during the first post-operative year. In contrast, gait speed and improved ability to climb stairs have been seen following bariatric surgery in as little as 3 months following the procedure [35].

\section{Outcomes of Orthopaedic surgery in morbidly obese patients}

Certainly, morbidly obese patients undergoing total joint replacement for OA present many challenge to the Orthopaedic surgeon and have higher rates of morbidity. On average, these patients are approximately 10 years younger than non-obese patients [36]. As the outcomes for total joint replacement are less favourable in younger patients in general, this potentiates the opportunity for early failure and a higher revision rate in this patient population. However, this has yet to be proven. During the operative procedure, technical difficulties are more likely to arise in the morbidly obese. The most common difficulty is in obtaining visualization and the lack of exposure. This may consequently lead to longer operative times, higher intra-operative blood loss, higher surgeon mental stress, and malposition of implants [37-39]. In addition, soft tissue damage to nerves, the collateral ligaments, and/or patella tendon avulsions are more likely [40].

Following the procedure, patients are found to have more complex medical management as a result of multiple medical comorbidities. They have a higher incidence of wound dehiscence, superficial infections, infections requiring surgical debridement, and thromboembolic events [41-43]. In fact, Baker et al. reported a 17\% wound complication rate in morbidly obese patients as compared to $9 \%$ in normal BMI individuals [44]. Patella-femoral pain is also found to be higher post-operatively in the obese group [45]. Also, due to a lack of overall body physical strength, rehabilitation is difficult. Furthermore, multiple studies have indicated a clear trend towards a higher rate of revision most often for septic and aseptic loosening in both total knee and total hip arthroplasties in morbidly obese patients [29,45-48]. For these reasons, it is beneficial for a patient to both lose and retain the weight loss prior to any orthopaedic procedures in an attempt to reduce patient morbidity and improve overall outcomes.

\section{Outcomes of bariatric surgery prior to orthopaedic surgery}

As a direct result of effective weight-loss achieved by patients undergoing bariatric surgery, juxtaposed against the ineffective weightloss seen by conventional management, there has been an increased interest in the orthopaedic field of managing obese patients by means of bariatric surgery prior to a planned orthopaedic surgical intervention. In spite of this interest, very few current studies have examined the outcomes of this proposed preoperative bariatric surgery. Parvizi et al. [30] conducted a small study of 12 patients who underwent knee or hip surgery within 24 months after either RYGB or VBG. Although the sample size was small and there lacked a control group, patients were found to have a significant improvement in pain scores, while only one patient required a revision of their arthroplasty. The lack of a control group was overlooked as the authors stated that these patients would not have been acceptable surgical candidates prior to bariatric surgery, as their obesity was considered a surgical contraindication [30]. Severson et al. investigated TKA preformed before bariatric surgery (group 1), within 2 years of bariatric surgery (group 2), 2 years following bariatric surgery (group 3) [49]. Their results showed significantly shorter anesthesia, operative, and tourniquet times in group 3 [49]. Thus, there seems to be a benefit to having patients undergo bariatric surgery prior to arthroplasty. The early success of this two-step bariatric-orthopaedic intervention warrants further study by means of controlled trials.

\section{Conclusion}

The complex relationship between obesity and osteoarthritis continues to require further investigations to be fully elucidated. Although many studies have been performed to give insight into this relationship, several important factors remain overlooked. Obesity appears to be directly related to pathological joint changes leading to both pain and osteoarthritis. This effect is due not only to increased mechanical load, but also to physiologic, humoral and inflammatory factors. Furthermore, the psychosomatic component of joint pain has not yet been explored. . The fact that obese individuals have a higher frequency of musculoskeletal pain than non-obese individuals opens the possibility that a psychosomatic component may play a large role $[8,28]$.

Bariatric surgery does seem to improve symptoms of osteoarthritis. This is felt to be true of both weight-bearing joints and non-weight bearing joints. Undoubtedly, factors other than mechanical load must play a part in the development of osteoarthritis. A small discordance that may explain some of the mixed results from different studies is the fact that OA was found to be significantly increased in patients with a BMI of over 30. However, bariatric surgery is indicated for patients with a BMI of over 40 or a BMI of over 35 with a corresponding obesity-related co morbidity. Weight-loss in many of these patients will be successful but will frequently not attain a level that is lower than a BMI of 30. This theoretically should not provide much benefit to the improvement of osteoarthritis in these patients. Further controlled trials are needed to clearly establish the effect of bariatric surgery on the progression of osteoarthritis.

An area that remains relatively unexplored is the relationship between bariatric surgery and the outcomes of subsequent arthroplasty. There are very few studies in this field, which is an area that may have large implications in the management strategy of these patients. The potential benefits that bariatric surgery prior to arthroplasty could range from eliminating the need for arthroplasty all-together, to dramatically improving post-operative outcomes for those who do undergo arthroplasty. Further trials in this area may pave the way for multi-modal management of these complex patients.

\section{Author contributions}

Lan $\mathrm{Vu}$ was involved in the drafting, research, editing and review of this manuscript. Noah Switzer was involved in the drafting, research, editing and review of this manuscript. D'Arcy Durand and Douglas Hedden was involved in the drafting, research, editing and review of this manuscript. Shahzeer Karmali was involved in the editing, review and final approval of this manuscript.

\section{References}

1. Buchwald H, Ikramuddin S, Dorman RB, Schone JL, Dixon JB (2011) Management of the metabolic/bariatric surgery patient. Am J Med 124: 10991105.

2. Maggard MA, Shugarman LR, Suttorp M, Maglione $M$, Sugerman $H J$, et al (2005) Meta-analysis: surgical treatment of obesity. Ann Intern Med 142: $547-$ 559. 
Citation: Vu L, Switzer NJ, Durand DAC, Hedden D, Karmali S (2013) Outcomes of Osteoarthritis after Bariatric Surgery. Surgery Curr Res 3: 141. doi:10.4172/2161-1076.1000141

Page 4 of 5

3. Buchwald $H$, Avidor $Y$, Braunwald $E$, Jensen MD, Pories W, et al. (2004) Bariatric surgery: a systematic review and meta-analysis. JAMA 292: 1724 1737.

4. Grundy SM, JI Cleeman, SR Daniels, KA Donato, RH Eckel, et al. (2005) American Heart, L. National Heart, and I. Blood. Diagnosis and management of the metabolic syndrome: an American Heart Association/National Heart, Lung, and Blood Institute Scientific Statement. Circulation 112:2735-2752.

5. Gill RS, Birch DW, Shi X, Sharma AM, Karmali S (2010) Sleeve gastrectomy and type 2 diabetes mellitus: a systematic review. Surg Obes Relat Dis 6: 707713.

6. Sarkhosh K, Birch DW, Shi X, Gill RS, Karmali S (2012) The impact of sleeve gastrectomy on hypertension: a systematic review. Obes Surg 22: 832-837.

7. Gill RS, Al-Adra DP, Shi X, Sharma AM, Birch DW, et al. (2011) The benefits of bariatric surgery in obese patients with hip and knee osteoarthritis: a systematic review. Obes Rev 12: 1083-1089.

8. Hooper MM, Stellato TA, Hallowell PT, Seitz BA, Moskowitz RW (2007) Musculoskeletal findings in obese subjects before and after weight loss following bariatric surgery. Int J Obes (Lond) 31: 114-120.

9. Felson DT (1990) The epidemiology of knee osteoarthritis: results from the Framingham Osteoarthritis Study. Semin Arthritis Rheum 20: 42-50.

10. Blagojevic M, Jinks C, Jeffery A, Jordan KP (2010) Risk factors for onse of osteoarthritis of the knee in older adults: a systematic review and metaanalysis. Osteoarthritis Cartilage 18: 24-33.

11. Lee R, Kean WF (2012) Obesity and knee osteoarthritis. Inflammopharmacology 20: 53-58.

12. Toivanen AT, Heliövaara M, Impivaara O, Arokoski JP, Knekt P, et al. (2010) Obesity, physically demanding work and traumatic knee injury are major risk factors for knee osteoarthritis--a population-based study with a follow-up of 22 years. Rheumatology (Oxford) 49: 308-314.

13. Andersen RE, Crespo CJ, Bartlett SJ, Bathon JM, Fontaine KR (2003) Relationship between body weight gain and significant knee, hip, and back pain in older Americans. Obes Res 11: 1159-1162.

14. Holmberg S, Thelin A, Thelin N (2005) Knee osteoarthritis and body mass index: a population-based case-control study. Scand J Rheumatol 34: 59-64

15. Melissas J, Volakakis E, Hadjipavlou A (2003) Low-back pain in morbidly obese patients and the effect of weight loss following surgery. Obes Surg 13: 389-393.

16. Schrager MA, Metter EJ, Simonsick E, Ble A, Bandinelli S, et al. (2007) Sarcopenic obesity and inflammation in the InCHIANTI study. J Appl Physiol 102: 919-925.

17. Vincent HK, Heywood K, Connelly J, Hurley RW (2012) Obesity and weight loss in the treatment and prevention of osteoarthritis. PM R 4: S59-67.

18. Sridhar MS, Jarrett CD, Xerogeanes JW, Labib SA (2012) Obesity and symptomatic osteoarthritis of the knee. J Bone Joint Surg Br 94: 433-440.

19. McNulty AL, Miller MR, O'Connor SK, Guilak F (2011) The effects of adipokines on cartilage and meniscus catabolism. Connect Tissue Res 52: 523-533.

20. Christensen R, Astrup A, Bliddal H (2005) Weight loss: the treatment of choice for knee osteoarthritis? A randomized trial. Osteoarthritis Cartilage 13: 20-27.

21. Messier SP, Loeser RF, Miller GD, Morgan TM, Rejeski WJ, et al. (2004) Exercise and dietary weight loss in overweight and obese older adults with knee osteoarthritis: the Arthritis, Diet, and Activity Promotion Trial. Arthritis Rheum 50: 1501-1510.

22. Gursoy A, Erdogan MF, Cin MO, Cesur M, Baskal N (2006) Comparison of orlistat and sibutramine in an obesity management program: efficacy, compliance, and weight regain after noncompliance. Eat Weight Disord 11 e127-132.

23. Brosseau L, Wells GA, Tugwell P, Egan M, Dubouloz CJ, et al. (2011) Ottawa Panel evidence-based clinical practice guidelines for the management of osteoarthritis in adults who are obese or overweight. Phys Ther 91: 843-861.

24. McGoey BV, Deitel M, Saplys RJ, Kliman ME (1990) Effect of weight loss on musculoskeletal pain in the morbidly obese. J Bone Joint Surg $\mathrm{Br} 72$ : 322-323.

25. Peltonen M, Lindroos AK, Torgerson JS (2003) Musculoskeletal pain in the obese: a comparison with a general population and long-term changes after conventional and surgical obesity treatment. Pain 104: 549-557.

26. Korenkov M, Shah S, Sauerland S, Duenschede F, Junginger T (2007) Impact of laparoscopic adjustable gastric banding on obesity co-morbidities in the medium- and long-term. Obes Surg 17: 679-683.

27. Abu-Abeid S, Wishnitzer N, Szold A, Liebergall M, Manor O (2005) The influence of surgically-induced weight loss on the knee joint. Obes Surg 15 : 1437-1442.

28. Roelofs J, Goubert L, Peters ML, Vlaeyen JW, Crombez G (2004) The Tampa Scale for Kinesiophobia: further examination of psychometric properties in patients with chronic low back pain and fibromyalgia. Eur J Pain 8: 495-502.

29. Foran JR, Mont MA, Etienne G, Jones LC, Hungerford DS (2004) The outcome of total knee arthroplasty in obese patients. J Bone Joint Surg Am 86-86A: 1609-15.

30. Parvizi J, Trousdale RT, Sarr MG (2000) Total joint arthroplasty in patients surgically treated for morbid obesity. J Arthroplasty 15: 1003-1008.

31. Dowsey MM, Liew D, Stoney JD, Choong PF (2010) The impact of preoperative obesity on weight change and outcome in total knee replacement: a prospective study of 529 consecutive patients. J Bone Joint Surg $\mathrm{Br}$ 92: 513520.

32. Howarth D, Inman D, Lingard E, McCaskie A, Gerrand C (2010) Barriers to weight loss in obese patients with knee osteoarthritis. Ann R Coll Surg Eng 92: 338-340.

33. Woodruff MJ, Stone MH (2001) Comparison of weight changes after total hip or knee arthroplasty. J Arthroplasty 16: 22-24.

34. Lachiewicz AM, Lachiewicz PF (2008) Weight and activity change in overweight and obese patients after primary total knee arthroplasty. J Arthroplasty 23: 3340.

35. Vincent HK, Ben-David K, Conrad BP, Lamb KM, Seay AN, et al. (2012) Rapid changes in gait, musculoskeletal pain, and quality of life after bariatric surgery. Surg Obes Relat Dis 8: 346-354.

36. Bourne R, Mukhi S, Zhu N, Keresteci M, Marin M (2007) Role of obesity on the risk for total hip or knee arthroplasty. Clin Orthop Relat Res 465: 185-188.

37. Prospective Studies Collaboration, Whitlock G, Lewington S, Sherliker $P$ Clarke R, et al. (2009) Body-mass index and cause-specific mortality in 900 000 adults: collaborative analyses of 57 prospective studies. Lancet 373: 10831096

38. Perka C, Labs K, Muschik M, Buttgereit F (2000) The influence of obesity on perioperative morbidity and mortality in revision total hip arthroplasty. Arch Orthop Trauma Surg 120: 267-271.

39. Gillespie GN, Porteous AJ (2007) Obesity and knee arthroplasty. Knee 14: 81 86.

40. Winiarsky R, Barth P, Lotke P (1998) Total knee arthroplasty in morbidly obese patients. J Bone Joint Surg Am 80: 1770-1774.

41. McCalden RW, Charron KD, MacDonald SJ, Bourne RB, Naudie DD (2011) Does morbid obesity affect the outcome of total hip replacement?: an analysis of 3290 THRs. J Bone Joint Surg Br 93: 321-325.

42. Guss D, Bhattacharyya T (2006) Perioperative management of the obese orthopaedic patient. J Am Acad Orthop Surg 14: 425-432.

43. Mantilla CB, Horlocker TT, Schroeder DR, Berry DJ, Brown DL (2003) Risk factors for clinically relevant pulmonary embolism and deep venous thrombosis in patients undergoing primary hip or knee arthroplasty. Anesthesiology 99 552-560.

44. Baker P, Petheram T, Jameson S, Reed M, Gregg P, et al. (2012) The association between body mass index and the outcomes of total knee arthroplasty. J Bone Joint Surg Am 94: 1501-1508.

45. Kerkhoffs GM, Servien E, Dunn W, Dahm D, Bramer JA, et al. (2012) The influence of obesity on the complication rate and outcome of total knee arthroplasty: a meta-analysis and systematic literature review. J Bone Joint Surg Am 94: 1839-1844

46. Jackson MP, Sexton SA, Yeung E, Walter WL, Walter WK, et al. (2009) The effect of obesity on the mid-term survival and clinical outcome of cementless total hip replacement. J Bone Joint Surg Br 91: 1296-1300.

47. Vazquez-Vela Johnson G, Worland RL, Keenan J, Norambuena N (2003) Patient demographics as a predictor of the ten-year survival rate in primary total knee replacement. J Bone Joint Surg Br 85: 52-56. 
Citation: Vu L, Switzer NJ, Durand DAC, Hedden D, Karmali S (2013) Outcomes of Osteoarthritis after Bariatric Surgery. Surgery Curr Res 3: 141. doi:10.4172/2161-1076.1000141

Page 5 of 5

48. Foran JR, Mont MA, Rajadhyaksha AD, Jones LC, Etienne G, et al. (2004) Total knee arthroplasty in obese patients: a comparison with a matched control group. J Arthroplasty 19: 817-824.
49. Severson EP, Singh JA, Browne JA, Trousdale RT, Sarr MG, et al. (2012) Total knee arthroplasty in morbidly obese patients treated with bariatric surgery: a comparative study. J Arthroplasty 27: 1696-1700. 